\title{
Terminal-Dependent Statistical Inference for the FBSDEs Models
}

\author{
Yunquan Song ${ }^{1,2}$ \\ ${ }^{1}$ China University of Petroleum, Qingdao 266580, China \\ ${ }^{2}$ Shandong University Qilu Securities Institute for Financial Studies, Shandong University, Jinan 250100, China
}

Correspondence should be addressed to Yunquan Song; math1212@163.com

Received 12 March 2014; Accepted 27 May 2014; Published 25 June 2014

Academic Editor: Guangchen Wang

Copyright (C) 2014 Yunquan Song. This is an open access article distributed under the Creative Commons Attribution License, which permits unrestricted use, distribution, and reproduction in any medium, provided the original work is properly cited.

The original stochastic differential equations (OSDEs) and forward-backward stochastic differential equations (FBSDEs) are often used to model complex dynamic process that arise in financial, ecological, and many other areas. The main difference between OSDEs and FBSDEs is that the latter is designed to depend on a terminal condition, which is a key factor in some financial and ecological circumstances. It is interesting but challenging to estimate FBSDE parameters from noisy data and the terminal condition. However, to the best of our knowledge, the terminal-dependent statistical inference for such a model has not been explored in the existing literature. We proposed a nonparametric terminal control variables estimation method to address this problem. The reason why we use the terminal control variables is that the newly proposed inference procedures inherit the terminal-dependent characteristic. Through this new proposed method, the estimators of the functional coefficients of the FBSDEs model are obtained. The asymptotic properties of the estimators are also discussed. Simulation studies show that the proposed method gives satisfying estimates for the FBSDE parameters from noisy data and the terminal condition. A simulation is performed to test the feasibility of our method.

\section{Introduction}

Since 1973, when the world's first options exchange opened in Chicago, a large number of new financial products have been introduced to meet the customer's demands from the derivative markets. In the same year, Black and Scholes [1] provided their celebrated formula for option pricing and Merton [2] proposed a general equilibrium model for security prices. Since then, modern finance has adopted stochastic differential equations as its basic instruments for portfolio management, asset pricing, risk management, and so on. Among these models, the backward stochastic differential equations (BSDEs for short) are a desirable choice for hedging and pricing an option. Its general form is as follows:

$$
\begin{gathered}
d Y_{s}=-g\left(s, Y_{s}, Z_{s}\right) d s+Z_{s} d B_{s}, \\
Y_{T}=\xi, \quad s \in[t, T],
\end{gathered}
$$

where $g$ is the generator, $B_{t}$ is a Brownian motion, and $\xi$ is a $\mathfrak{R}$-valued Borel function as the terminal condition. Usually the terminal condition is designed as a random variable with given distribution. If $g$ meets certain conditions, the BSDE has a unique solution.

In terms of the backward equation, within a complete market, it serves to characterize the dynamic value of replicating portfolio $Y_{s}$ with a final wealth $\xi$ and a special quantity $Z_{s}$ that depends on the hedging portfolio. In particular, while the generator consists of diffusion process, the corresponding equation is proved to be a forward-backward stochastic differential equation (FBSDE), which can be expressed as

$$
d Y_{s}=-g\left(s, X_{s}, Y_{s}, Z_{s}\right) d s+Z_{s} d B_{s}, \quad Y_{T}=\xi,
$$

where $X_{s}$ satisfies the following ordinary stochastic differential equation (OSDE):

$$
d X_{s}=\mu\left(s, X_{s}\right) d t+\sigma\left(s, X_{s}\right) d B_{s}, \quad s \in[t, T] .
$$

Compared to the OSDE that contains an initial condition, the solution of the FBSDE is affected by the terminal condition $Y_{T}=\xi\left(X_{T}\right)$. As is well known, there exist a number 
of parametric and nonparametric methods to deal with estimation and test for the OSDE. However, these methods cannot be directly employed to infer the BSDE and FBSDE because the two models are related to a terminal condition. Forward-backward stochastic differential equations are used in biology systems, mathematical finance, insurance, real estate, multiagent, and network control. See Antonelli [3], Wang et al. [4], Zhang and $\mathrm{Li}$ [5], and so on.

For the FBSDE defined above, the statistical inference was investigated initially by Su and Lin [6] and Chen and Lin [7]. Furthermore, by financial and ecological problems, a relevant statistical model was proposed by Lin et al. [8]. However, they did not take the terminal condition into account in the inference procedure. In the framework of the FBSDE mentioned above, the terminal condition is additional, which is not nested into the equation. Thus, there is an essential difficulty to use the terminal condition to refine the inference procedure.

As a result, their methods fail to cover the full problems given in the FBSDE. Zhang and Lin [9] proposed two terminal-dependent estimation methods via terminal control variable for the integral form models of FBSDE. However, they only considered the parametric form of the generator $g$ in their paper.

This paper intends to explore the method to fulfill the terminal-dependent inference: quasi-instrumental variable methods. It is worth mentioning that the key point of our method is the use of the terminal condition information rather than neglecting it. This change leads to a completely new work among the existing researches. The key technique in our method is the use of quasi-instrumental variable which is similar but not the same as instrumental variable (IV). It is known that IV is widely employed in applied econometrics to achieve identification and carry out estimation and inference in the model containing endogenous explanatory variables or panel data; see Hsiao [10] for an overview of the relevant statistical inference and econometric interpretation and see Hall and Horowitz [11] for recent work on nonparametric instrumental variable estimation.

Through the backward equation (2) of FBSDE, we get a regression model. To use the terminal condition information, we put the terminal condition as a quasi-instrumental variable and introduce it into our model. However, when a constraint is appended artificially, the original model may change to be biased in the sense of $E\left(Z_{s} d B_{s} \mid X_{s}, \xi\right) \neq 0$, because the constraint condition influences the increase trend of wealth so that $Z_{s} d B_{s}$ may deviate from the original center zero; in other words, due to the constraint, the trajectory of $Y_{s}$ may departure from the original expectation so that $Z_{s} d B_{s}$ cannot be regarded as error. Therefore, some problems arise naturally, including how to correct the bias of the model and how to construct the constraint-dependent estimation. To solve these problems, we will use remodeling method to draw terminal condition into differential equation, similar but not the same as IV, called quasi-instrumental variable methods; in other words, the terminal condition $\xi$ enters into the equation as a control variable. This remodeling method takes advantage of the terminal information naturally, and the estimator performs quite well.
We use the nonparametric form of the generator $g$ in model (2) because the correct FBSDEs model for a specific topic can neither be provided automatically by financial market nor be derived from theory of mathematical finance, and in lack of prior information about the structure of a model, nonparametric inference can provide a flexible as well as robust description of a data-generating process. Even in some cases when parametric models are available, nonparametric methods are still employed to avoid the model misspecification that may lead to large errors in option pricing and other problems from financial market. So we adopt the nonparametric form that can endow the model (2) with flexibility and robustness.

Note that $Z_{s}$ is usually unobservable and $g$ cannot be completely specified in the financial market. The problems of interest are therefore to give both proper estimations of the generator $g$ and the process $Z_{s}$ based on the observed data $\left(X_{s}, Y_{s}\right)$ and the terminal expectation $\xi$.

The remainder of the paper is organized as follows. In Section 2, the FBSDE is rebuilt as a nonparametric model that contains the terminal condition as a quasi-instrumental variable. Consequently, a terminal-dependent estimation procedure is proposed. Next we discuss the asymptotic properties of the newly proposed estimations in Section 3. Simulation study is proposed in Section 4 to illustrate our methods. The proofs of the theorems are presented in Appendix.

\section{Model and Method}

In this section, we propose a nonparametric estimator with the help of quasi-instrumental variable.

2.1. Model and Its Statistical Version. We begin the following original model by combining (2)-(3):

$$
\begin{aligned}
& d Y_{s}=-g\left(s, X_{s}, Y_{s}, Z_{s}\right) d s+Z_{s} d B_{s}, \quad Y_{T}=\xi, \\
& d X_{s}=\mu\left(s, X_{s}\right) d t+\sigma\left(s, X_{s}\right) d B_{s}, \quad s \in[t, T],
\end{aligned}
$$

where $B_{t}$ is the standard Brownian motion and $\xi$ is a $\mathfrak{R}$ valued Borel function. Here the generator $g$ is a function of $s, X_{s}, Y_{s}$, and $Z_{s}$. For the FBSDEs model (4), only one of the backward components, $Y_{s}$, and the forward components, $X_{s}$, can be observed. Another backward component $Z_{s}$ is totally unobservable. Furthermore, the adapted process $Z_{s}$ and terminal condition could be indicated as a function of $X_{s}$.

In this section, we present the statistical structure of FBSDEs by taking advantage of quasi-instrumental variable and obtain the consistent asymptotically normal estimators of $g$ and $Z_{s}$ based on observed data $\left\{X_{s}, Y_{s}\right\}$ and the terminal condition $\xi$.

2.2. Remodeling for Model (4). To construct terminaldependent estimation for the generator $g$ and process $Z_{s}$, the key technique is how to plug the terminal condition into the equation. When $\xi$ is plugged into the model, we call it the quasi-IV, similar but not the same as IV. Evidently, the property of Brownian motion shows that $E\left(Z_{s} d B_{s} \mid X_{s}\right)=0$, 
but $E\left(Z_{s} d B_{s} \mid X_{s}, \xi\right) \neq 0$, which means drawing the terminal control directly into the equation as the condition should not be encouraged at the cost of model bias. Rewriting the first equation of (4) enables us to construct an unbiased model:

$$
d Y_{s}=-g\left(s, X_{s}, Y_{s}, Z_{s}\right) d s+m\left(X_{s}, \xi\right)+U_{s},
$$

where $m\left(X_{s}, \xi\right)=E\left(Z_{s} d B_{s} \mid X_{s}, \xi\right), U_{s}=Z_{s} d B_{s}-$ $m\left(X_{s}, \xi\right)$, and $E\left(U_{s} \mid X_{s}, \xi\right)=0$. The newly defined model (5), together with the second equation in (4), can be thought of as a quasi-IV FBSDE. Because the equation in (5) contains the terminal condition $\xi$, we can construct the terminal-dependent estimation. From the above definitions, we see that, by bias correction, the original model changes to be an additive nonparametric model with nonparametric components $-g\left(s, X_{s}, Y_{s}, Z_{s}\right) d s$ and $m\left(X_{s}, \xi\right)$. It shows that when terminal condition is regarded as a quasi-IV and then appended to the model, the result model is unbiased and changes to be nonparametric additive model.

2.3. Estimation for $Z_{s}$. Before estimating the model function $m\left(x_{s}, \xi\right)$ and the generator $g$, we need to estimate $Z_{s}$ firstly because $Z_{s}$ is unobservable and it will be seen that the estimators of the model function $m\left(x_{s}, \xi\right)$ and the generator $g$ depend on $Z_{s}$. Since the distribution of $\xi$ is supposed to be known, let $\left\{\xi_{i}, 1 \leq i \leq k\right\}$ for $k \geq 1 / \Delta$ be a sample of $\xi$. Suppose that, for each terminal data $\xi_{j}$ and equally spaced time points $\left\{s_{i}=s_{1}+(i-1) \Delta, i=1, \ldots, n\right\} \subseteq[0, T]$, we record the observed time series data:

$$
\begin{aligned}
& \left\{X_{s_{i}, j}, Y_{s_{i}, j}, i=1, \ldots, n, j=1, \ldots, k\right\} \\
& \quad=\left\{X_{i, j}, Y_{i, j}, i=1, \ldots, n, j=1, \ldots, k\right\} .
\end{aligned}
$$

At any time point $s \in[t, T], Z_{s}^{t, x}$, denoting $Z_{s}$ and satisfying the initial condition $(t, x)$, is a determined function of $X_{s}^{t, x}$. As was shown by Su and Lin [6] and Chen and Lin [7], we can adopt a difference-based method to approximate $Z^{2}$ as

$$
\left(Z_{s}^{t, X_{t}}\right)^{2}=\frac{1}{\Delta} E\left(Y_{s+\Delta}^{t+\Delta, X_{t}+\Delta}-Y_{s}^{t, X_{t}} \mid X_{t}, t\right)^{2}+O(\Delta)
$$

It shows that the numerical approximation error to $Z_{t}^{2}$ converges to zero at rate of order $O_{p}(\Delta)$.

For each $\xi_{j}$, if $Z_{t}$ depends on $t$ only via variable $X_{t}$, by (7) and N-W kernel estimation method, we estimate $Z_{t}^{2}$ at $x_{0}$ by

$$
\widehat{Z}_{x_{0}, j}^{2}=\frac{\sum_{i=1}^{n-1} \Delta^{-1}\left(Y_{i+1, j}-Y_{i, j}\right)^{2} K_{h_{X}}\left(X_{i, j}-x_{0}\right)}{\sum_{i=1}^{n-1} K_{h_{X}}\left(X_{i, j}-x_{0}\right)} .
$$

Otherwise, we estimate $Z_{t}^{2}$ at $\left(x_{0}, t_{0}\right)$ by

$$
\begin{aligned}
& \widehat{Z}_{x_{0}, t_{0}, j}^{2} \\
& \quad=\frac{\sum_{i=1}^{n-1} \Delta^{-1}\left(Y_{i+1, j}-Y_{i, j}\right)^{2} K_{h_{X}}\left(X_{i, j}-x_{0}\right) K_{h_{t}}\left(t_{i}-t_{0}\right)}{\sum_{i=1}^{n-1} K_{h_{X}}\left(X_{i, j}-x_{0}\right) K_{h_{t}}\left(t_{i}-t_{0}\right)},
\end{aligned}
$$

where $K_{h_{X}}=K\left(\cdot / h_{X}\right) / h_{X}$ and $K_{h_{t}}=K\left(\cdot / h_{t}\right) / h_{t}, K(\cdot)$ are regular kernel functions, with $h_{X}$ and $h_{t}$ being the corresponding bandwidths.
2.4. Estimation for $m\left(X_{s}, \xi\right)$. After plugging the estimator $\widehat{Z}_{s}$ into model (5), we still need to consider inference of $m\left(x_{s}, \xi\right)$. As we all know, the nonparametric function $m\left(X_{s}, \xi\right)$ in (5) can be acquired as $m\left(X_{s}, \xi\right)=E\left(d Y_{s}+g\left(s, X_{s}, Y_{s}, Z_{s}\right) d s\right.$ । $\left.X_{s}, \xi\right)$. We note that $g\left(s, X_{s}, Y_{s}, Z_{s}\right) d s$ is a higher order infinitesimal of $Z_{s} d B_{s}$ when $\Delta$ tends to zero. Under this situation, if $g\left(s, X_{s}, Y_{s}, Z_{s}\right) d s$ is ignored, then

$$
m\left(X_{s}, \xi\right) \doteq E\left(d Y_{s} \mid X_{s}, \xi\right) \text {. }
$$

It implies that we can use ordinary nonparametric method to estimate function $m$. For example, we use the N-W ordinary nonparametric method to estimate $m\left(X_{s}, \xi\right)$ valued at $\left(x_{0}, \xi_{0}\right)$ :

$$
\begin{aligned}
& \widehat{m}\left(x_{0}, \xi_{0}\right) \\
& =\frac{\sum_{i=1}^{n-1} \sum_{j=1}^{m}\left(Y_{i+1, j}-Y_{i, j}\right) K_{h_{X}}\left(X_{i, j}-x_{0}\right) K_{h_{\xi}}\left(\xi_{i, j}-\xi_{0}\right)}{\sum_{i=1}^{n-1} \sum_{j=1}^{m} K_{h_{X}}\left(X_{i, j}-x_{0}\right) K_{h_{\xi}}\left(\xi_{i, j}-\xi_{0}\right)},
\end{aligned}
$$

where $K_{h_{X}}=K\left(\cdot / h_{X}\right) / h_{X}$ and $K_{h_{\xi}}=K\left(\cdot / h_{\xi}\right) / h_{\xi}$ are regular kernel functions, with $h_{X}$ and $h_{\xi}$ being the corresponding bandwidths.

2.5. Estimation for Generator g. As was shown in the nonparametric instrumental variables estimator of Hall and Horowitz [11] (hereinafter $\mathrm{HH}$ ), we can adopt a nonparametric quasi-instrumental variables estimation to estimate the generator $g$. So in the section we summarize the $\mathrm{HH}$ estimator of $g$ in the model:

$$
E\left[-d Y_{t}-m\left(X_{t}, \xi\right) \mid X_{t}, \xi\right]=E\left[g\left(t, X_{t}, Y_{t}, Z_{t}\right) d t \mid X_{t}, \xi\right]
$$

Since $\widehat{m}\left(x_{0}, \xi_{0}\right)$ and $\widehat{Z}_{x_{0}, j}^{2}$ are the consistent estimator of $m\left(x_{0}, \xi_{0}\right)$ and $Z_{x_{0}, j}^{2}$, respectively, we use them instead of $m\left(X_{s}, \xi\right)$ and $Z_{s}$ in the above model and we get

$$
\begin{aligned}
E & {\left[-d Y_{s}-\widehat{m}\left(X_{s}, \xi\right) \mid X_{s}, \xi\right] } \\
& =E\left[g\left(s, X_{s}, Y_{s}, \widehat{Z}_{s}\right) d t \mid X_{s}, \xi\right] .
\end{aligned}
$$

Because $\widehat{Z}_{s}$ is function of $X_{s}$ and $Y_{s}$, for simplicity of presentation, we denote $g\left(s, X_{s}, Y_{s}, \widehat{Z}_{s}\right)=g\left(X_{s}, Y_{s}\right)$. Thus, the model becomes

$$
E\left[-d Y_{s}-\widehat{m}\left(X_{s}, \xi\right) \mid X_{s}, \xi\right]=E\left[g\left(X_{s}, Y_{s}\right) d t \mid X_{s}, \xi\right]
$$

Let $\mathbb{Y}_{i}=\left(\left(Y_{i+\Delta}-Y_{i}\right)-\widehat{m}\left(X_{i}, \xi\right)\right) / \Delta, \mathbb{X}_{i}=X_{i}, \mathbb{Z}_{i}=Y_{i}$, $\mathbb{W}=\xi$, and $\mathbb{U}_{i}=V_{i} / \sqrt{\Delta}$; the model becomes

$$
\mathbb{Y}_{i}=g\left(\mathbb{X}_{i}, \mathbb{Z}_{i}\right)+\mathbb{U}_{i}, \quad E\left(\mathbb{U}_{i} \mid \mathbb{X}_{i}, \mathbb{W}_{i}\right)=0 .
$$

It is assumed that the support of $(\mathbb{X}, \mathbb{Z}, \mathbb{W})$ is contained in $[0,1]^{3}$. This assumption can always be satisfied by, if necessary, carrying out monotone increasing transformations of $\mathbb{X}, \mathbb{Z}$, and $\mathbb{W}$. For example, one can replace $\mathbb{X}, \mathbb{Z}$, and $\mathbb{W}$ by 
$\Phi(\mathbb{X}), \Phi(\mathbb{Z})$, and $\Phi(\mathbb{W})$, where $\Phi$ is the normal distribution function. We take $(\mathbb{Y}, \mathbb{X}, \mathbb{Z}, \mathbb{W}, \mathbb{U})$ to be a vector, where $\mathbb{Y}$ and $\mathbb{U}$ are scalars, $\mathbb{X}$ and $\mathbb{W}$ are supported on $[0,1]$, and $\mathbb{Z}$ is supported on $[0,1]$. The model is

$$
\mathbb{Y}_{i}=g\left(\mathbb{X}_{i}, \mathbb{Z}_{i}\right)+\mathbb{U}_{i}, \quad E\left(\mathbb{U}_{i} \mid \mathbb{Z}_{i}, \mathbb{W}_{i}\right)=0,
$$

where $\left(\mathbb{Y}_{i}, \mathbb{X}_{i}, \mathbb{Z}_{i}, \mathbb{W}_{i}, \mathbb{U}_{i}\right)$, for $i \geq 1$, are independent and identically distributed as $(\mathbb{Y}, \mathbb{X}, \mathbb{Z}, \mathbb{W}, \mathbb{U})$. Thus, $\mathbb{X}$ and $\mathbb{Z}$ are endogenous and exogenous explanatory variables, respectively. Data $\left(\mathbb{Y}_{i}, \mathbb{X}_{i}, \mathbb{Z}_{i}, \mathbb{W}_{i}, \mathbb{U}_{i}\right)$, for $1 \leq i \leq n$, are observed.

Let $f_{\mathbb{X} \mathbb{Z} W}$ denote the density of $(\mathbb{X}, \mathbb{Z}, \mathbb{W})$, write $f_{\mathbb{Z}}$ for the density of $\mathbb{Z}$, and, for each $x_{1}, x_{2} \in[0,1]^{p}$, and put

$$
t_{z}\left(x_{1}, x_{2}\right)=\int f_{\mathbb{X} \mathbb{Z} \mathbb{W}}\left(x_{1}, z, w\right) f_{\mathbb{X} \mathbb{W}}\left(x_{2}, z, w\right) d w .
$$

Define the operator $T_{z}$ on $L_{2}[0,1]^{p}$ by

$$
\left(T_{z} \psi\right)(x)=\int t_{z}(\xi, x) \psi(\xi) d \xi
$$

It may be proved that, for each $z$ for which $T_{z}^{-1}$ exists,

$$
\begin{aligned}
& g(x, z) \\
& =f_{\mathbb{Z}}(z) E_{\mathbb{W} \mid \mathbb{Z}} \\
& \quad \times\left\{E(\mathbb{Y} \mid \mathbb{Z}=z, \mathbb{W})\left(T_{z}^{-1} f_{\mathbb{X} \mathbb{W}}\right)(x, z, \mathbb{W}) \mid \mathbb{Z}=z\right\},
\end{aligned}
$$

where $E_{\mathbb{W} \mid \mathbb{Z}}$ denotes the expectation with respect to the distribution of $\mathbb{W}$ conditional on $\mathbb{Z}$. In this formulation, $\left(T_{z}^{-1} f_{\mathbb{X} \mathbb{Z} W}\right)(x, z, \mathbb{W})$ denoted the result of applying $T_{z}^{-1}$ to the function $f_{\mathbb{X} \mathbb{Z} \mathbb{W}}(\cdot, z, \mathbb{W})$ and evaluating the resulting function at $x$.

To construct an estimator of $g(x, z)$, given $h>0$ and $x=$ $x^{(1)}$ and $\xi=\xi^{(1)}$, let $K_{h}(x, \xi)=K_{h}\left(x^{(j)}, \xi^{(j)}\right)$, put $K_{h}(z, \xi)$ analogously for $z$ and $\xi$, let $h_{x}, h_{z}>0$, and define

$$
\begin{aligned}
& \widehat{f}_{\mathbb{X} \mathbb{Z} W}(x, z, w) \\
& =\frac{1}{n h_{x}^{2} h_{z}} \sum_{i=1}^{n} K_{h_{x}}\left(x-\mathbb{X}_{i}, x\right) K_{h_{z}}\left(z-\mathbb{Z}_{i}, z\right) K_{h_{x}}\left(w-\mathbb{W}_{i}, w\right), \\
& \widehat{f}_{\mathbb{X} \mathbb{Z} \mathbb{W}}^{-i}(x, z, w) \\
& =\frac{1}{(n-1) h_{x}^{2} h_{z}} \sum_{1 \leq j \leq n: j \neq i} K_{h_{x}}\left(x-\mathbb{X}_{j}, x\right) \\
& \quad \times K_{h_{z}}\left(z-\mathbb{Z}_{j}, z\right) K_{h_{z}}\left(w-\mathbb{W}_{j}, w\right), \\
& \quad\left(\widehat{T}_{z} \psi\right)(x, z, w)=\int \widehat{t}_{z}(\xi, x) \psi(\xi, z, w) d \xi,
\end{aligned}
$$

where $\psi$ is a function from $R^{3}$ to a real line. Then the estimator of $g(x, z)$ is

$$
\widehat{g}(x, z)=\frac{1}{n} \sum_{i=1}^{n}\left(\widehat{T}_{z}^{+} \widehat{f}_{\mathbb{X} \mathbb{Z} \mathbb{W}}^{-i}\right)\left(x, z, \mathbb{W}_{i}\right) Y_{i} K_{h_{z}}\left(z-\mathbb{Z}_{i}, z\right) .
$$

\section{Asymptotic Results}

In this section, we study the asymptotic properties of our proposed estimators. All proofs are presented in Appendix.

3.1. Asymptotic results of $\widehat{Z}_{s}$. To give the asymptotic results of $\widehat{Z}_{s}$, we need the following conditions.

(a) $X_{1}, \ldots, X_{n}$ are $\rho$-mixing dependent; namely, the $\rho$ mixing coefficients $\rho(l)$ satisfy $\rho(l) \rightarrow 0$ as $l \rightarrow \infty$, where

$\rho(l)=\sup _{E\left(X_{i+l} X_{i}\right)-E\left(X_{i+l}\right) E\left(X_{i}\right) \neq 0} \frac{\left|E\left(X_{i+l} X_{i}\right)-E\left(X_{i+l}\right) E\left(X_{i}\right)\right|}{\sqrt{\operatorname{Var}\left(X_{i+l}\right) \operatorname{Var}\left(X_{i}\right)}}$

with $X_{i}=X\left(t_{i}\right)$.

(b) $\left|Z_{i}\right| \leq C$ (a. s.) uniformly for $i=1, \ldots, n$, where $C$ is a positive constant and $Z_{i}=Z\left(t_{i}\right)$.

(c) The continuous kernel function $K(\cdot)$ is symmetric about 0 , with a support of interval $[-1,1]$, and

$$
\begin{gathered}
\int_{-1}^{1} K(u) d u=1, \quad \sigma_{K}^{2}=\int_{-1}^{1} u^{2} K(u) d u \neq 0 \\
\int_{-1}^{1}|u|^{j} K^{k}(u) d u<\infty \quad \text { for } j \leq k=1,2 .
\end{gathered}
$$

Condition (a) is commonly used for weakly dependent process; see, for example, Kolmogorov and Rozanov [12], Bradley and Bryc [13], Lu and Lin [14], and Su and Lin [6]. Condition (b) is also reasonable because, as is shown by (10), $Z_{t}$ can be regarded as the deviation between the adjacent two observations. Condition (c) is standard for nonparametric kernel function.

Theorem 1. Besides conditions (a), (b), and (c), let $\left\{X_{1}, \ldots, X_{n}\right\}$ be an observation sequence on a stationary $\rho$-mixing Markov process with the $\rho$-mixing coefficients satisfying $\rho(l)=\rho^{l}$ for $0<\rho<1$. Furthermore, $X_{1}, \ldots, X_{n}$ have a common and probability density $p(x)$, and for each interior point $x_{0}$ in the support of $p(\cdot), p\left(x_{0}\right)>0, Z^{2}\left(x_{0}\right)>0$, the functions $p(x)$ and $Z(x)$ have continuous two derivatives in neighborhood of $x_{0}$. As $n \rightarrow \infty$, such that $n h \rightarrow \infty$, $n h^{5} \rightarrow 0$, and $n h \Delta^{2} \rightarrow 0$, then

$$
\sqrt{n h}\left(\widehat{Z}^{2}\left(x_{0}\right)-Z^{2}\left(x_{0}\right)\right) \stackrel{d}{\longrightarrow}\left(0, \frac{Z^{4}\left(x_{0}\right) J_{K}}{p\left(x_{0}\right)}\right),
$$

where $J_{K}=\int_{-1}^{1} K^{2}(u) d u<\infty$.

The asymptotic result in Theorem 1 is standard for nonparametric kernel estimator and here undersmoothing is used to eliminate asymptotic bias.

3.2. Asymptotic results of $\widehat{g}(x, z)$. This section gives conditions under which the $\mathrm{HH}$ estimator of the generator 
$g$ is asymptotically distributed as $N(0, I)$. The following additional notations are used.

Define $\mathbb{U}_{i}=\mathbb{Y}_{i}-g\left(\mathbb{X}_{i}, \mathbb{Z}_{i}\right), S_{n 1}(x, z)=$ $n^{-1} \sum_{i=1}^{n} \mathbb{U}_{i} \widehat{T}^{+} \widehat{f}_{\mathbb{X} \mathbb{Z} W}^{(-i)}\left(x, z, \mathbb{W}_{i}\right) K_{q, h_{z}}\left(z-\mathbb{Z}_{i}, z\right)$, and $S_{n 2}(x, z)=$ $n^{-1} \sum_{i=1}^{n} g\left(\mathbb{X}_{i}, \mathbb{Z}_{i}\right) \widehat{T}^{+} \widehat{f}_{\mathbb{X} \mathbb{Z} \mathbb{W}}^{(-i)}\left(x, z, \mathbb{W}_{i}\right) K_{q, h_{z}}\left(z-\mathbb{Z}_{i}, z\right)$. Then, $\widehat{g}(x, z)=S_{n 1}(x, z)+S_{n 2}(x, z)$. Define $T^{+}=\left(T+a_{n} I\right)^{-1}$. Write

$$
\begin{aligned}
& S_{n 1}(x, z) \\
& =n^{-1} \sum_{i=1}^{n} \mathbb{U}_{i}\left(T^{+} f_{\mathbb{X} \mathbb{Z} \mathbb{W}}\right)\left(x, z, \mathbb{W}_{i}\right) K_{q, h_{z}}\left(z-\mathbb{Z}_{i}, z\right) \\
& +n^{-1} \sum_{i=1}^{n} \mathbb{U}_{i}\left(\widehat{T}^{+} \widehat{f}_{\mathbb{X} \mathbb{Z} \mathbb{W}}^{(-i)}-T^{+} f_{\mathbb{X} \mathbb{Z} \mathbb{W}}\right) \\
& \quad \times\left(x, z, \mathbb{W}_{i}\right) K_{q, h_{z}}\left(z-\mathbb{Z}_{i}, z\right) \\
& =S_{n 11}(x, z)+S_{n 12}(x, z) .
\end{aligned}
$$

Define $V_{n}(x, z)=n^{-1} \operatorname{Var}\left[\mathbb{U}\left(T^{+} f_{\mathbb{X} \mathbb{Z} \mathbb{W}}\right)(x, z, \mathbb{W})\right]$. It follows from a triangular array version of the Lindeberg-Levy central limit theorem that $S_{n 11}(x, z) / \sqrt{V_{n}(x, z)} \rightarrow{ }^{d} N(0,1)$ as $n \rightarrow$ $\infty$. Therefore, $[\hat{g}(x, z)-g(x, z)] / \sqrt{V_{n}(x, z)} \rightarrow{ }^{d} N(0,1)$ if $\left[S_{n 12}(x, z)+S_{n 2}(x, z)-g(x, z)\right] / \sqrt{V_{n}(x, z)}=o_{p}(1)$.

Assumption 2. The data $\mathbb{Y}_{i}, \mathbb{X}_{i}, \mathbb{Z}_{i}, \mathbb{W}_{i}$ are independently and identically distributed as $(\mathbb{Y}, \mathbb{X}, \mathbb{X}, \mathbb{W})$, where $(\mathbb{X}, \mathbb{Z}, \mathbb{W})$ is supported on $[0,1]^{3}$ and $E[\mathbb{Y}-g(\mathbb{X}, \mathbb{Z}) \mid \mathbb{W}, \mathbb{Z}]=0$

Assumption 3. The distribution of $(\mathbb{X}, \mathbb{Z}, \mathbb{W})$ has a density $f_{\mathbb{X} \mathbb{Z} W}$ with respect to Lebesgue measure. Moreover, $f_{\mathbb{X} \mathbb{Z} W}$ is $r$ times differentiable with respect to any combination of its arguments, where derivatives at the boundary of $[0,1]^{3}$ are defined as one sided derivatives. The derivatives are bounded in absolute value by $C$. In addition, $g$ is $r$ times differentiable on $[0,1]^{2}$ with derivatives at 0 and 1 defined as one sided. The derivatives of $g$ are bounded in absolute value by $C$. In addition, $E\left[\mathbb{Y}^{2} \mid \mathbb{X}, \mathbb{Z}, \mathbb{W}\right] \leq C$ and $E\left[\mathbb{Y}^{2} \mid \mathbb{X}, \mathbb{Z}, \mathbb{W}\right] \leq C$, and $E\left[\mathbb{U}^{2} \mid \mathbb{Z}, \mathbb{W}\right] \geq C_{U}$ for some finite constant $C_{U}$.

Assumption 4. The constants $\alpha$ and $\beta$ satisfy $\alpha>1, \beta>1 / 2$, and $\beta-1 / 2 \leq \alpha<2 \beta$. Moreover, $b_{j} \leq C j^{-\beta}, j^{-\alpha} \leq C \lambda_{j}$, and $\sum_{k=1}^{\infty}\left|d_{z j k}\right| \leq C j^{-\alpha / 2}$ for all $j \geq 1$. In addition, there are finite strictly positive constants, $C_{\lambda_{1}}$ and $C_{\lambda 2}$, such that $C_{\lambda_{1}} \leq \lambda_{j} \leq$ $C_{\lambda 2} j^{-\alpha}$ for all $j \geq 1$.

Assumption 5. The tuning parameters $a_{n}$ and $h$ satisfy $a_{n} \asymp$ $n^{-(\rho \alpha) /(2 \beta+\alpha)}$ and $h=n^{-1}$, where $r \in\left[A_{2}^{\prime}, A_{3}^{\prime}\right]$.

Assumption 6. $K_{h}$ denotes a generalized kernel function, with the properties $K_{h}(u, t)=0$ if $u>t$ or $u<t-1$, for all $t \in[0,1] h^{-(j+1)} \int_{t-1} t u^{j} K_{h}(u, t) d u=1$ if $j=0$, else 0 if $1 \leq j \leq r-1$. For each $\xi \in[0,1], K_{h}(h, \xi)$ is supported on $[(\xi-1) / h, \xi / h] \cap \kappa$, where $\kappa$ is a compact interval not depending on $\xi$. Moreover,

$$
\sup _{h>0, \xi \in[0,1], u \in \kappa} K_{h}(h u, \xi) \mid<\infty .
$$

Assumption 7. Consider $E_{\mathbb{W}}\left[T^{+} f_{\mathbb{X} \mathbb{Z} W}(x, z, \mathbb{W})\right]^{2}=$ $E_{\mathbb{W}}\left[T^{+} f_{\mathbb{X} \mathbb{Z} \mathbb{W}}(\cdot, \cdot, \mathbb{W})\right]^{2} \quad$ and $\quad E_{\mathbb{W}}\left[T^{+} f_{\mathbb{X} \mathbb{Z} \mathbb{W}}(\cdot, \cdot, \mathbb{W})\right]^{2}=$ $\int_{0}^{1}\left\|T^{+} f_{\mathbb{X} \mathbb{Z} W}(\cdot, \cdot, \mathbb{W})\right\|^{2} d w$.

Theorem 8. Let Assumptions 2-7 hold. Then

$$
\frac{\widehat{g}(x, z)-g(x, z)}{\sqrt{V_{n}(x, z)}} \rightarrow^{d} N(0, I)
$$

holds except, possibly, on a set of $(x, z)$ values whose Lebesgue is 0 .

Corollary 9. Let Assumptions 2-7 hold. And if $V_{n}(x, z)$ is replaced with the consistent estimator,

$$
\widehat{V}_{n}(x, z)=n^{-1} \sum_{i=1}^{n} \widehat{\mathbb{U}}_{i}^{2}\left[\widehat{T}^{+} \widehat{f}_{x w}^{-i}\left(z, \mathbb{W}_{i}\right) K_{q, h_{z}}\left(z-\mathbb{Z}_{i}, z\right)\right]^{2},
$$

where $\widehat{\mathbb{U}}_{i}=\mathbb{Y}_{i}-\widehat{g}\left(\mathbb{X}_{i}, \mathbb{Z}_{i}\right)$. This yields the Studentized statistic $[\widehat{g}(x, z)-g(x, z)] / \sqrt{\widehat{V}_{n}(x, z)}$. Then

$$
\frac{\widehat{g}(x, z)-g(x, z)}{\sqrt{\widehat{V}_{n}(x, z)}} \rightarrow{ }^{d} N(0, I)
$$

holds except, possibly, on a set of $(x, z)$ values whose Lebesgue is 0 .

As was shown in the remark given in the previous section, even the conditional mean of error of the model is nonzero, and the newly proposed estimation is consistent because of the mixing dependency; for details see the proof of Theorem 8. Furthermore, because of the terminal condition, the asymptotic variance is larger than that without the use of the terminal condition.

\section{Simulation Studies}

In this section, we investigate the finite-sample behaviors by simulation.

Example 10. We consider a simple FBSDE as

$$
\begin{aligned}
d Y_{t} & =\left(\frac{\mu-r}{\sigma} Z_{t}+r Y_{t}\right) d t+Z_{t} d B_{t} \\
& \triangleq\left(b Y_{t}+c Z_{t}\right)+Z_{t} d B_{t} ; \quad Y_{T}=\xi
\end{aligned}
$$

where $X_{t}$ is Geometric Brownian motion for modeling stock price satisfying

$$
d X_{t}=\mu X_{t} d t+\sigma X_{t} d B_{t} ; \quad X_{0}=x,
$$

while the riskless asset is the same as formula (31); $d P_{t}=$ $r P_{0} d t$.

Firstly, let $\mu=0.1, \sigma=0.01, \Delta=0.12, n=300$, $T=36.6$, and $n_{0}=n_{1}=10$. Obviously, $Z_{t}=n_{1} \sigma X_{t}$. We adopt Epanechnikov kernel defined by $K(u)=3 / 4\left(1-u^{2}\right) I(|u| \leq 1)$, 


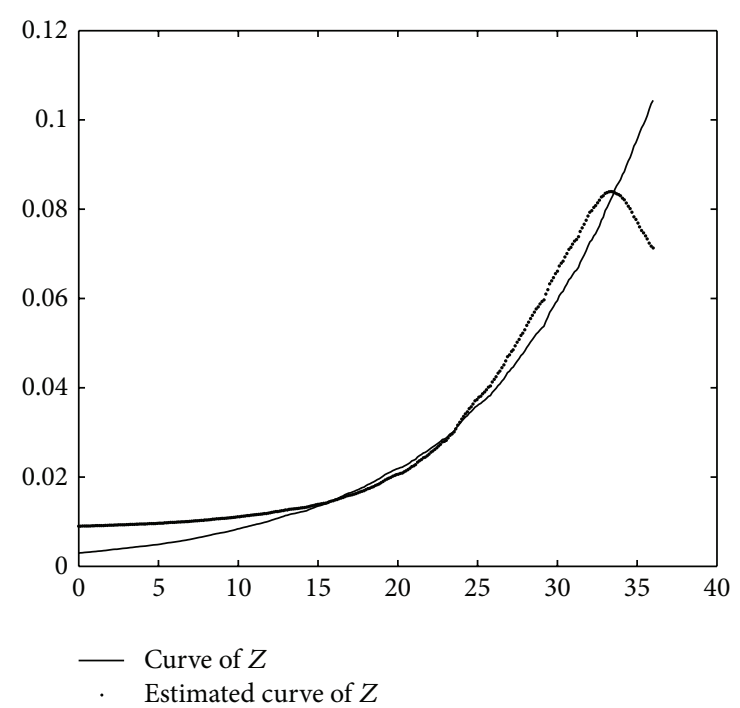

(a)

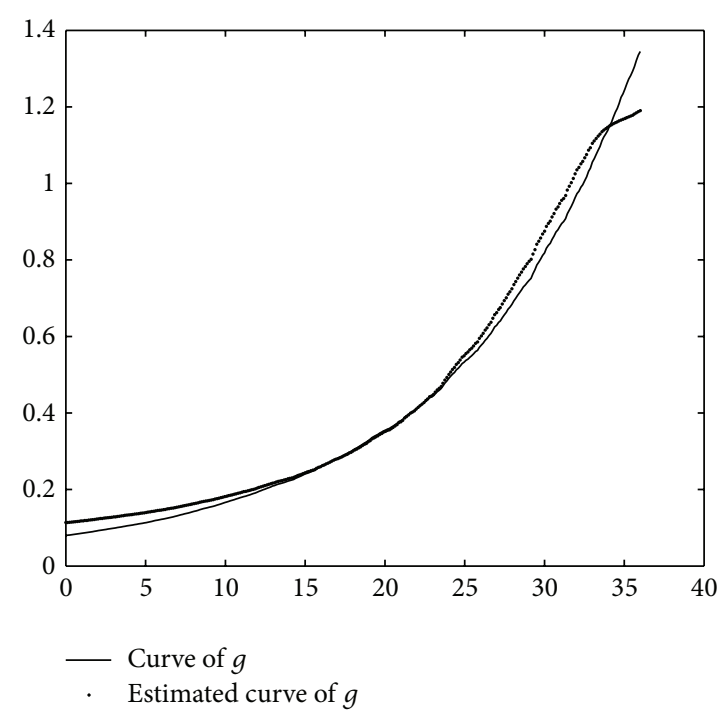

(b)

Figure 1: The real lines are the true curves of $Z_{t}$ and function $g(t)$, respectively, and the dashed ones are estimated curves for them in Example 10 .

where $I(\cdot)$ is the indicator function. For bandwidth selection, various data-driven techniques have been developed, such as cross-validation, the plug-in method, and the empirical bias method. However, these useful tools require additional computation intensiveness. In our simulation, we simply apply the rule of thumb bandwidth selector. For bandwidth selection, bandwidth $h=\operatorname{std}(x) n^{-1 / 5}$. The values of the tuning parameters are $a_{n}=0.05, \alpha=1.2, \beta=1$. Figure 1 presents the estimated curves for diffusion $Z_{t}$ and drift $g$ by one simulation.

Example 11. According to the theory of mathematical finance, we represent a European call option by the following FBSDEs model:

$$
\begin{gathered}
d X_{s}=b X_{s} d s+\sigma X_{s} d W_{s}, \\
d Y_{s}=\left[r Y_{s}+(b-r) \sigma^{-1} Z_{s}\right] d s+Z_{s} d W_{s}, \\
X_{0}=x, \quad Y_{T}=\left(X_{T}-K\right)^{+}, \quad s \in[0, T] .
\end{gathered}
$$

Here $\left\{X_{s}\right\}_{0 \leq s \leq T}$ and $\left\{Y_{s}\right\}_{0 \leq s \leq T}$ are the price processes of the stock and the option, respectively, and $K$ is the striking price at the expiration date T. $\left\{X_{s}\right\}_{0 \leq s \leq T}$ follows the geometric Brownian motion as

$$
\begin{gathered}
d X_{s}=b X_{s} d s+\sigma X_{s} d W_{s}, \\
X_{0}=x, \quad s \in[0, T] .
\end{gathered}
$$

We use the Euler scheme to generate the price series of the stock as

$$
X_{i+1}-X_{i}=b X_{i} \Delta+\sigma X_{i} \Delta^{1 / 2} \epsilon_{i}, \quad i=0, \ldots, n-1,
$$

where $\left\{\epsilon_{i}\right\}_{i=0}^{n-1}$ is an i.i.d. series with standard normality.
The price series by Black Scholes formula is part of the solution of the FBSDEs above at discrete time points; that is,

$$
Y_{i}=X_{i} N\left(d_{+}^{i}\right)-e^{-r(n-i) \Delta} K N\left(d_{-}^{i}\right),
$$

which, together with

$$
Z_{i}=\sigma X_{i} N\left(d_{+}^{i}\right),
$$

gives us data generating formulae, where

$$
N(y)=\frac{1}{\sqrt{2 \pi}} \int_{-\infty}^{y} e^{-x^{2} / 2} d x
$$

is a cumulative normal function, and

$$
d_{ \pm}^{i}=\frac{\ln \left(X_{i} / K\right)+\left(r \pm \sigma^{2} / 2\right)((n-i) \Delta)}{\sigma \sqrt{(n-i) \Delta}} .
$$

We produce the true curve of the drift coefficient by

$$
g_{i}=-r Y_{i}-(b-r) \sigma^{-1} Z_{i} .
$$

We first apply formulas (21) and (11) to estimate $g_{i}$ and $Z_{i}^{2}$, respectively. We adopt Epanechnikov kernel defined by $K(u)=3 / 4\left(1-u^{2}\right) I(|u| \leq 1)$, where $I(\cdot)$ is the indicator function. For bandwidth selection, we simply apply the rule of thumb bandwidth selector:

$$
h=\text { constant } \times \operatorname{std}\left(Y_{0}, \ldots, Y_{n-1}\right) n^{-1 / 5}
$$

to implement the estimation.

Let $K=110, X_{0}=100, b=0.1, \sigma=0.18, r=0.08$, $T=60$, and $\Delta=1 / 100$. The bandwidth parameters $h=6.06$ and $h=0.67$ are used for estimation of $g_{s}$ and $Z_{s}$, respectively. The values of the tuning parameters are $a_{n}=0.05, \alpha=1.2$, and $\beta=1$. To see the performance of our estimation method, the simulated and the estimated curves of the two coefficients of the backward equation are displayed in Figures 2 and 3. 


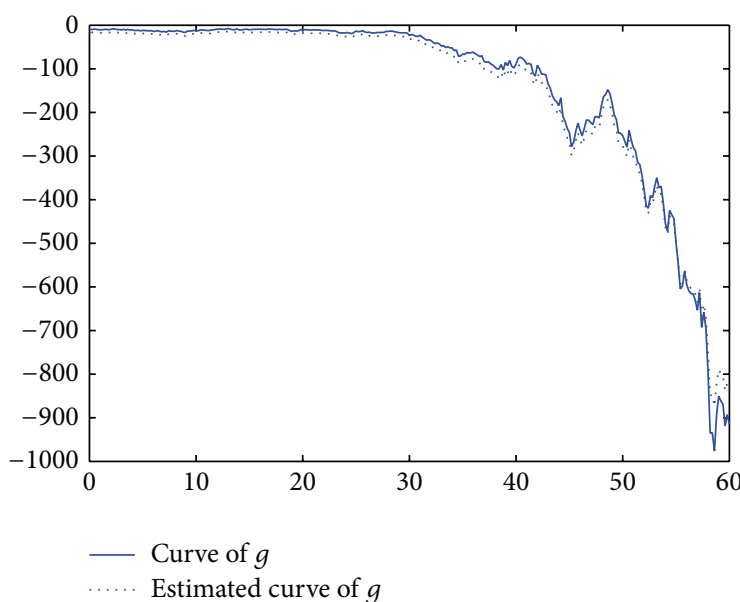

FIGURE 2: The simulated curve and the estimated curves of $g_{s}$ in Example 11.

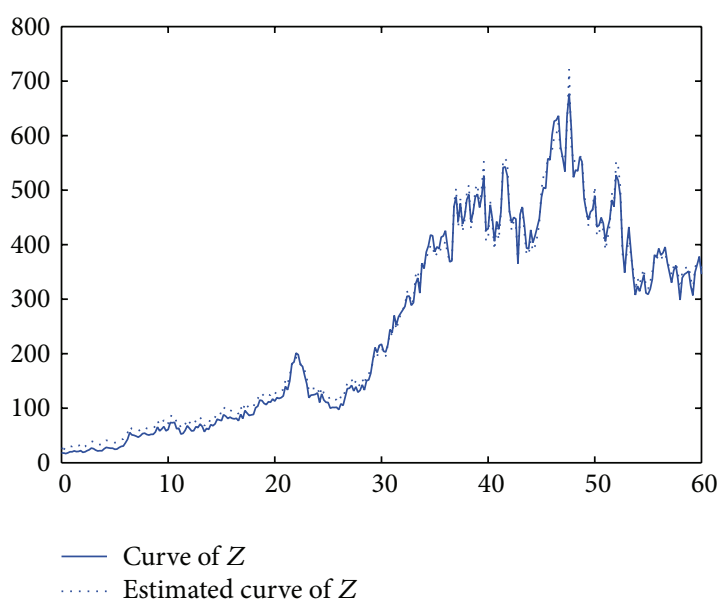

FIGURE 3: The simulated curve and the estimated curves of $Z_{s}$ in Example 11.

\section{Appendix}

\section{A. Proofs}

Proof of Theorem 1. Denote $\mathscr{C}=\left\{X_{1}, \ldots, X_{n}, \ldots\right\}$. By the Taylor expansion and formula (8), we have

$$
\begin{aligned}
E & \left(\widehat{Z}^{2}\left(x_{0}\right) \mid \mathscr{C}\right) \\
& =\frac{\sum_{i=1}^{n-1} \Delta^{-1} K_{h}\left(X_{i}-x_{0}\right) E\left(\left(Y_{i+1}-Y_{i}\right)^{2} \mid \mathscr{C}\right)}{\sum_{i=1}^{n-1} K_{h}\left(X_{i}-x_{0}\right)} \\
& =\frac{\sum_{i=1}^{n-1} K_{h}\left(X_{i}-x_{0}\right)\left(Z_{i}^{2}+O(\Delta)\right)}{\sum_{i=1}^{n-1} K_{h}\left(X_{i}-x_{0}\right)} \\
& =\frac{\int K_{h}\left(X_{i}-x_{0}\right)\left(Z^{2}(x)+O(\Delta)\right) p(x) d x\left(1+O_{p}(n h)^{-1 / 2}\right)}{\int K_{h}\left(X_{i}-x_{0}\right) p(x) d x\left(1+O_{p}(n h)^{-1 / 2}\right)}
\end{aligned}
$$

$$
\begin{aligned}
&=(\left(Z^{2}\left(x_{0}\right)+O(\Delta)\right) \\
& \times\left(p\left(x_{0}\right)+(1 / 2) h^{2} p^{(2)}\left(x_{0}\right) \sigma_{K}^{2}+o\left(h^{2}\right)\right) \\
&\left.\times\left(1+O_{p}(n h)^{-1 / 2}\right)\right) \\
& \times\left(\left(p\left(x_{0}\right)+(1 / 2) h^{2} p^{(2)}\left(x_{0}\right) \sigma_{K}^{2}+o\left(h^{2}\right)\right)\right. \\
&\left.\times\left(1+O_{p}(n h)^{-1 / 2}\right)\right)^{-1} \\
&=Z^{2}\left(x_{0}\right)+\frac{p^{(2)}\left(x_{0}\right)}{2 p\left(x_{0}\right)} h^{2} Z^{2}\left(x_{0}\right) \sigma_{K}^{2}+o\left(h^{2}\right)+O(\Delta) .
\end{aligned}
$$

Furthermore,

$$
\begin{aligned}
& \operatorname{Var}\left(\widehat{Z}^{2}\left(x_{0}\right) \mid \mathscr{C}\right) \\
&= \frac{1}{\sum_{i=1}^{n-1} K_{h}^{2}\left(X_{i}-x_{0}\right)} \\
& \times\left\{\sum_{i=1}^{n-1} \Delta^{-2} K_{h}^{2}\left(X_{i}-x_{0}\right) \operatorname{Var}\left(\left(Y_{i+1}-Y_{i}\right)^{2} \mid \mathscr{C}\right)\right. \\
&+\sum_{i=1}^{n-1} \sum_{k=1}^{n-i} \Delta^{-2} \operatorname{cov}\left(K_{h}\left(X_{i}-x_{0}\right)\left(Y_{i+1}-Y_{i}\right),\right. \\
&\left.\left.K_{h}\left(X_{i+k}-x_{0}\right)\left(Y_{i+k+1}-Y_{i+k}\right) \mid \mathscr{C}\right)\right\}
\end{aligned}
$$

From the conditions of Markov process and $\rho$-mixing coefficient,

$$
\begin{aligned}
& \mid \sum_{i=1}^{n-1} \sum_{k=1}^{n-i} \Delta^{-2} \operatorname{cov}\left(K_{h}\left(X_{i}-x_{0}\right)\left(Y_{i+1}-Y_{i}\right),\right. \\
& \left.\quad K_{h}\left(X_{i+k}-x_{0}\right)\left(Y_{i+k+1}-Y_{i+k}\right)\right) \mid \\
& =\frac{1}{(n-1)^{2}} \sum_{i=1}^{n-1} \sum_{k=1}^{n-i} \mid E\left((\Delta)^{-2}\left(Y_{i+1}-Y_{i}\right)^{2}\left(Y_{i+k+1}-Y_{i+k}\right)^{2}\right. \\
& \quad \times\left(K_{h}\left(X_{i}-x_{0}\right)-E\left(K_{h}\left(X_{i}-x_{0}\right)\right)\right) \\
& \left.=\frac{1}{(n-1)^{2}} \mid E\left(K_{h}\left(X_{i+k}-x_{0}\right)-E\left(K_{h}\left(X_{i+k}-x_{0}\right)\right)\right)\right) \mid \\
& \quad+O\left(K_{h}\left(X_{i}-x_{0}\right)-E\left(K_{h}\left(X_{i}-x_{0}\right)\right)\right) \\
& \leq \frac{C}{(n-1)^{2} h} \sum_{i=1}^{n-1} \sum_{k=1}^{n-i} \rho^{k}=O\left(\frac{1}{n h}\right)=o(1) .
\end{aligned}
$$


Note that $\left(Y_{i+1}-Y_{i}\right) / \sqrt{\Delta}=g\left(t_{i}, Y_{i}, Z_{i}\right) \sqrt{\Delta}+Z_{i} \eta_{i}$, where $E\left(\eta_{i}\right)=0, \operatorname{Var}\left(\eta_{i}\right)=1$. Thus $\operatorname{Var}\left(\left(Y_{i+1}-Y_{i}\right) / \sqrt{\Delta}\right)=Z_{i}^{4}+O(\Delta)$ and furthermore

$$
\begin{aligned}
\operatorname{Var} & \left(\widehat{Z}^{2}\left(x_{0}\right) \mid \mathscr{C}\right) \\
& =\frac{\sum_{i=1}^{n-1} \Delta^{-2} K_{h}^{2}\left(X_{i}-x_{0}\right) \operatorname{Var}\left(\left(Y_{i+1}-Y_{i}\right)^{2} \mid \mathscr{C}\right)}{\sum_{i=1}^{n-1} K_{h}^{2}\left(X_{i}-x_{0}\right)}+O_{p}(1) \\
& =\frac{\sum_{i=1}^{n-1} K_{h}^{2}\left(X_{i}-x_{0}\right)\left(Z^{4}\left(x_{0}\right)+O(\sqrt{\Delta})\right)}{\sum_{i=1}^{n-1} K_{h}^{2}\left(X_{i}-x_{0}\right)}+O_{p}(1) \\
& =\frac{Z^{4}\left(x_{0}\right) J_{K}+O(\sqrt{\Delta})}{n h p\left(x_{0}\right)}\left(1+O_{p}(n h)^{-1 / 2}\right) .
\end{aligned}
$$

To our interest, both the conditional expectation and variance are independent on $\mathscr{C}$, so the condition could be erased.

From Lemma A.1 of Politis and Romano [15] and the relation between the $\alpha$-mixing condition and the $\rho$-mixing condition (e.g., Theorem 1.1.1 of Lu and Lin [14]), we can ensure that $\left\{\left(Y_{i+1}-Y_{i}\right)^{2}, i=1, \ldots, n-1\right\}$ is a $\rho$-mixing dependent process and the mixing coefficient, denoted by $\rho_{Y}(l)$, satisfies

$$
\sum_{k=1}^{\infty} \rho_{Y}\left(2^{k}\right) \leq C \sum_{k=1}^{\infty} \rho\left(2^{k}\right)=\sum_{k=1}^{\infty} \rho^{2^{k}}<\infty,
$$

where $C$ is a positive constant. Finally, we use the central limit theorems for $\rho$-mixing dependent process (e.g., Theorem 4.0.1 of Lu and Lin [14]) to complete this proof.

Proof of Theorem 8. Theorem 8 follows from proving that $S_{n 1}(x, z) / \sqrt{V_{n}(x, z)} \rightarrow{ }^{d} N\left(0, I_{2}\right)$ and $\left[S_{n 2}(x, z)-g(x, z)\right] /$ $\sqrt{V_{n}(x, z)}=o_{p}(1)$ except, possibly, if $(x, z)$ belongs to a set of Lebesgue measure 0 . The first result is established in Lemma A.1, and the second is established in Lemma A.2. Throughout this Appendix, "for almost every $(x, z)$ " means "for every $(x, z) \in[0,1]^{2}$ except, possibly, a set of Lebesgue measure 0 ." We make repeated use of the fact that if $E\|\psi\|^{2}=$ $O\left(n^{-s}\right)$ for some $s>0$, then $\psi(x, z)=o_{p}\left(n^{-s}\right)$ for almost every $(x, z)$.

Lemma A.1 (asymptotic normality of $S_{n 1}(x, z) / \sqrt{V_{n}(x, z)}$ ). Let Assumptions 2-7 hold. Then $S_{n 1}(x, z) / \sqrt{V_{n}(x, z)} \rightarrow{ }^{d} N(0$, $I_{2}$ ) for almost every $(x, z)$.

Proof. Define $S_{n 11}(x, z)=n^{-1} \sum_{i=1}^{n} \mathbb{U}_{i}\left(T^{+} f_{\mathbb{X Z \mathbb { W }}}\right)\left(x, z, \mathbb{W}_{i}\right)$,

$$
\begin{aligned}
& A_{n 2}(x, z)
\end{aligned}
$$

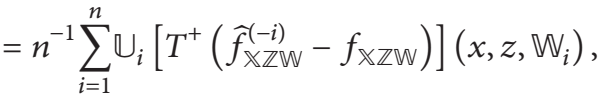

$$
\begin{aligned}
& A_{n 3}(x, z) \\
& =n^{-1} \sum_{i=1}^{n} \mathbb{U}_{i}\left[\left(\widehat{T}^{+}-T^{+}\right) f_{\nwarrow \mathbb{Z} \mathbb{W}}\right]\left(x, z, \mathbb{W}_{i}\right),
\end{aligned}
$$

$$
\begin{aligned}
& A_{n 4}(x, z) \\
& \quad=n^{-1} \sum_{i=1}^{n} \mathbb{U}_{i}\left[\left(\widehat{T}^{+}-T^{+}\right)\left(\widehat{f}_{\mathbb{X} \mathbb{Z} \mathbb{W}}^{(-i)}-f_{\mathbb{X} \mathbb{Z} W}\right)\right]\left(x, z, \mathbb{W}_{i}\right) .
\end{aligned}
$$

Then $S_{n 1}(x, z)=S_{n 11}(x, z)+A_{n 2}(x, z)+A_{n 3}(x, z)+A_{n 4}(x, z)$. $S_{n 11}(x, z) / \sqrt{V_{n}(x, z)} \rightarrow^{d} N\left(0, I_{2}\right)$ by a triangular array version of the Lindeberg-Levy central limit theorem. The proof of the triangular-array version of the theorem is identical to the proof of the ordinary Lindeberg-Levy theorem. The lemma follows if we can prove that $A_{n j}(x, z) / \sqrt{V_{n}(x, z)}=o_{p}(1)$ for $j=2,3,4$ and almost every $(x, z) \in[0,1]^{2}$.

Assumption 7 and arguments like those leading to (6.2) of $\mathrm{HH}[11]$ show that

$$
\iint_{0}^{1} V_{n}(x, z) d x d z \asymp n^{-[2 \beta+\alpha-\rho(\alpha+1)] /(2 \beta+\alpha)} .
$$

It follows from the Cauchy-Schwartz inequality, $E\left(\widehat{f}_{\mathbb{X} \mathbb{Z} W}^{(-i)}-\right.$ $\left.f_{\mathbb{X} \mathbb{W}}\right)=O\left(h^{\prime}\right)$, and $\operatorname{Var}\left(\widehat{f}_{\mathbb{X} \mathbb{Z} \mathbb{W}}^{(-i)}\right)=O\left[1 /\left(n h^{2}\right)\right]$ that

$$
E\left\|A_{n 2}\right\|^{2}=O\left(\frac{1}{n^{2} h^{2} a_{n}^{2}}+\frac{h^{2 r}}{n a_{n}^{2}}\right) .
$$

Therefore, it follows from Assumptions 5 and 7 that $A_{n 2}(x, z) / \sqrt{V_{n}(x, z)}=o_{p}(1)$ for almost every $(x, z)$. Now consider $A_{n 3}(x, z)$. Define the operator $\Delta=\widehat{T}-T$. Then

$$
A_{n 3}(x, z)=-\left(\widehat{T}+a_{n} I\right) \Delta A_{n 1}(x, z) .
$$

Therefore, the Cauchy-Schwartz inequality gives

$$
\begin{aligned}
E\left\|A_{n 2}\right\|^{2} & \leq E\left\|\left(\widehat{T}+a_{n} I\right) \Delta\right\|^{2} E\left\|S_{n 11}\right\|^{2} \\
& =E\left\|\left(\widehat{T}+a_{n} I\right) \Delta\right\|^{2} \iint_{0}^{1} V_{n}(x, z) d x d z .
\end{aligned}
$$

$\mathrm{HH}$ show that

$$
E\left\|\left(\widehat{T}+a_{n} I\right) \Delta\right\|^{2}=O\left(\frac{1}{n h a_{n}^{2}}+\frac{h^{2 r}}{a_{n}^{2}}\right) .
$$

Therefore, it follows from Assumptions 5 and 7 that $A_{n 3}(x, z) / \sqrt{V_{n}(x, z)}=o_{p}(1)$ for almost every $(x, z)$. Finally, some algebra shows that

$$
A_{n 4}(x, z)=-\left(\widehat{T}+a_{n} I\right)^{-1} \Delta A_{n 2}(x, z) .
$$

Therefore, $A_{n 4}(x, z) / \sqrt{V_{n}(x, z)}=o_{p}(1)$ for almost every $(x$, $z$ ) follows from (A.11) and $A_{n 2}(x, z) / \sqrt{V_{n}(x, z)}=o_{p}(1)$.

Lemma A.2 (asymptotic negligibility of $S_{n 2}(x, z)-g(x, z)$ ). Let Assumptions 2-7 hold. Then $S_{n 2}(x, z)-g(x, z) /$ $\sqrt{V_{n}(x, z)}=o_{p}(1)$ for almost every $(x, z)$. 
Proof. Define

$$
\begin{aligned}
& D_{n}(x, z)=\iiint_{0}^{1} g(\theta, \eta) f_{\mathbb{X} \mathbb{Z}}(\theta, \eta, w) T^{+} \\
& \quad \times\left(\widehat{f}_{\mathbb{X} \mathbb{Z} \mathbb{W}}-f_{\mathbb{X} \mathbb{Z} \mathbb{W}}\right)(x, z, w) d \theta d \eta d w, \\
& A_{n 1}(x, z)=n^{-1} \sum_{i=1}^{n} g\left(\mathbb{X}_{i}, Z_{i}\right)\left(T^{+} f_{\mathbb{X} \mathbb{Z} \mathbb{W}}\right)\left(x, z, \mathbb{W}_{i}\right) .
\end{aligned}
$$

Redefine

$$
\begin{aligned}
& A_{n 2}(x, z) \\
& =n^{-1} \sum_{i=1}^{n} g\left(\mathbb{X}_{i}, \mathbb{Z}_{i}\right)\left[T^{+}\left(\widehat{f}_{\mathbb{X} \mathbb{Z} \mathbb{W}}^{(-i)}-f_{\mathbb{X} \mathbb{Z} \mathbb{W}}\right)\right]\left(x, z, \mathbb{W}_{i}\right) \\
& -D_{n}(x, z), \\
& A_{n 3}(x, z) \\
& =n^{-1} \sum_{i=1}^{n} g\left(\mathbb{X}_{i}, \mathbb{Z}_{i}\right)\left[\left(\widehat{T}^{+}-T^{+}\right) f_{\mathbb{X} \mathbb{Z} \mathbb{W}}\right]\left(x, z, \mathbb{W}_{i}\right)+D_{n}(x, z), \\
& A_{n 4}(x, z) \\
& \begin{aligned}
=n^{-1} \sum_{i=1}^{n} g\left(\mathbb{X}_{i}, \mathbb{Z}_{i}\right)\left[\left(\widehat{T}^{+}-T^{+}\right)\left(\widehat{f}_{\mathbb{X} \mathbb{Z} \mathbb{W}}^{(-i)}-f_{\mathbb{X} \mathbb{Z} \mathbb{W}}\right)\right] \\
\times\left(x, z, \mathbb{W}_{i}\right) .
\end{aligned}
\end{aligned}
$$

Then $S_{n 2}(x, z)=\sum_{j=1}^{4} A_{n j}(x, z)$. Arguments identical to those used to derive (6.2) and (6.3) of HH [11] show that $\left\|E A_{n 1}-g\right\|^{2}=O\left[n^{-\rho\left(2 \beta_{1}\right) /(2 \beta+\alpha)}\right]$ and

$$
\iint_{0}^{1} \operatorname{Var}\left[A_{n 1}(x, z)\right] d x d z=O n^{-[2 \beta+\alpha-\rho(\alpha+1)] /(2 \beta+\alpha)} .
$$

Therefore, it follows from Assumptions 5 and 7 that

$$
\begin{gathered}
\frac{\left[E A_{n 1}(x, z)-g(x, z)\right]}{\sqrt{V_{n}(x, z)}}=o(1) \\
V_{n}^{-1}(x, z) \iint_{0}^{1} \operatorname{Var}\left[A_{n 1}(x, z)\right] d x d z=O(1)
\end{gathered}
$$

for almost every $(x, z)$.

Now consider $A_{n 2}(x, z)$. Define

$$
\begin{aligned}
D_{n i}(x, z)=\iiint_{0}^{1} g(\theta, \eta) f_{\mathbb{X} \mathbb{W}}(\theta, \eta, w) T^{+} \\
\times\left(\widehat{f}_{\mathbb{X} \mathbb{Z} \mathbb{W}}^{(-i)}-f_{\mathbb{X} \mathbb{Z} W}\right)(x, z, w) d \theta d \eta d w \\
A_{n 21}(x, z)=n^{-1} \sum_{i=1}^{n} g\left(\mathbb{X}_{i}, \mathbb{Z}_{i}\right)\left[T^{+}\left(\widehat{f}_{\mathbb{X} \mathbb{Z} \mathbb{W}}^{(-i)}-f_{\mathbb{X} \mathbb{W}}\right)\right] \\
\times\left(x, z, \mathbb{W}_{i}\right)-D_{n i}(x, z),
\end{aligned}
$$

and $A_{n 22}(x, z)=n^{-1} \sum_{i=1}^{n}\left[D_{n i}(x, z)-D_{n}(x, z)\right]$. HH show that $\left\|E A_{n 21}\right\|^{2}=O\left(\left(h^{2 r} / n a_{n}^{2}\right)+\left(1 / n^{2} h^{2} a_{n}^{2}\right)\right)$ and $\left\|E A_{n 22}\right\|^{2}=$ $\mathrm{O}\left(1 / n^{2} a_{n}^{2}\right)$. Therefore, it follows from Assumptions 5 and 7 that

$$
\frac{A_{n 2}(x, z)}{\sqrt{V_{n}(x, z)}}=o_{p}(1)
$$

for almost every $(x, z)$. Now consider $A_{n 3}(x, z)$. Write

$$
A_{n 3}(x, z)=A_{n 31}(x, z)+A_{n 32}(x, z)
$$

where $A_{n 31}(x, z)=-\left(I+T^{+} \Delta\right)^{-1} T^{+} \Delta g(x, z)+D_{n}(x, z)$ and $A_{n 32}(x, z)=-\left(\widehat{T}^{+}+a_{n} I\right)^{-1} \Delta\left(A_{n 1}-g\right)(x, z)$. It follows from (A.11)-(A.16) and (A.20) that

$$
\frac{A_{n 32}(x, z)}{\sqrt{V_{n}(x, z)}}=o_{p}(1)
$$

for almost every $(x, z)$.

To analyze $A_{n 31}(x, z)$, define

$$
\begin{aligned}
& B_{n 1}(x, z)=\iiint_{0}^{1}\left[\widehat{f}_{\mathbb{X} \mathbb{Z W}}(x, z, w)-f_{\mathbb{X} \mathbb{Z W}}(x, z, w)\right] \\
& \times f_{\mathbb{X} \mathbb{Z W}}(x, z, w) g(x, z) d x d z d w, \\
& B_{n 2}(x, z)=\iiint_{0}^{1}\left[\widehat{f}_{\mathbb{X} \mathbb{Z W}}(x, z, w)-f_{\nwarrow \mathbb{Z} \mathbb{W}}(x, z, w)\right] \\
& \times f_{\mathbb{X} \mathbb{Z} W}(x, x, w) g(x, x) d x d z d w, \\
& B_{n 3}(x, z)=\iiint_{0}^{1}\left[\widehat{f}_{\mathbb{X} \mathbb{W}}(x, z, w)-f_{\mathbb{X} \mathbb{Z W}}(x, z, w),\right. \\
& \left.\widehat{f}_{\mathbb{X} \mathbb{Z} W}(x, z, w)-f_{\mathbb{X} \mathbb{Z W}}(x, z, w)\right] \\
& \times g(x, z) d x d z d w
\end{aligned}
$$

$$
\begin{aligned}
B_{n 11}(x, z)=\iiint_{0}^{1}\left[E \widehat{f}_{\mathbb{X} \mathbb{Z} W}(x, z, w)-f_{\mathbb{X} \mathbb{Z W}}(x, z, w)\right] \\
\times f_{\mathbb{X} \mathbb{Z} W}(x, z, w) g(x, z) d x d z d w, \\
B_{n 12}(x, z)=\iiint_{0}^{1}\left[\widehat{f}_{\mathbb{X} \mathbb{Z} W}(x, z, w)-E \widehat{f}_{\mathbb{X} \mathbb{Z} W}(x, w)\right] \\
\times f_{\mathbb{X} \mathbb{Z} W}(x, z, w) g(x, z) d x d z d w,
\end{aligned}
$$

$$
\begin{aligned}
B_{n 21}(x, z)=\iiint_{0}^{1} & {\left[E \widehat{f}_{\mathbb{X} \mathbb{Z W}}(x, z, w)-f_{\mathbb{X} \mathbb{Z W}}(x, z, w)\right] } \\
& \times f_{\mathbb{X} \mathbb{Z} W}(x, z, w) g(x, z) d x d z d w
\end{aligned}
$$

$$
\begin{aligned}
B_{n 22}(x, z)=\iiint_{0}^{1} & {\left[\widehat{f}_{\mathbb{X} \mathbb{Z} \mathbb{W}}(x, z, w)-E \widehat{f}_{\mathbb{X} \mathbb{Z} W}(x, z, w)\right] } \\
& \times f_{\mathbb{X} \mathbb{Z} W}(x, z, w) g(x, z) d x d z d w .
\end{aligned}
$$


Define $\delta=h^{2 r}+(n h)^{-1}$. HH show that

$$
\begin{aligned}
A_{n 31}(x, z)= & -\left(I+T^{+} \Delta\right)^{-1} T^{+}\left(B_{n 11}+B_{n 12}+B_{n 3}\right)(x, z) \\
& +\left(I+T^{+} \Delta\right)^{-1} T^{+} \Delta T^{+}\left(B_{n 21}+B_{n 22}\right)(x, z) .
\end{aligned}
$$

Define

$$
\begin{aligned}
\widetilde{A}_{n 31}(x, z)= & -\left(I+T^{+} \Delta\right)^{-1} T^{+}\left(B_{n 11}+B_{n 12}+B_{n 3}\right)(x, z) \\
& +\left(I+T^{+} \Delta\right)^{-1} T^{+} \Delta T^{+} B_{n 21} .
\end{aligned}
$$

Then

$$
\begin{gathered}
E\left\|A_{n 31}\right\|^{2} \\
\leq \text { const. }\left[E\left\|\widetilde{A}_{n 31}\right\|^{2}+E\left\|(I+T \Delta)^{-1} T^{+} \Delta T^{+} B_{n 22}\right\|^{2}\right] \\
E\left\|\widetilde{A}_{n 31}\right\|^{2} \leq \text { const. }\left(\left\|T^{+} B_{n 11}\right\|^{4}+E\left\|T^{+} B_{n 12}\right\|^{4}\right. \\
\left.+E\left\|T^{+} \Delta T^{+} B_{n 21}\right\|^{4}+E\left\|T^{+} B_{n 3}\right\|^{4}\right)^{1 / 2} .
\end{gathered}
$$

$\mathrm{HH}$ show that

$$
\begin{gathered}
\left\|T^{+} B_{n 11}\right\|=O\left(\frac{h^{r}}{a_{n}}\right), \\
\left(E\left\|T^{+} \Delta T^{+} B_{n 21}\right\|^{4}\right)^{1 / 2}=O\left(\frac{\delta h^{2 r}}{a_{n}}\right), \\
\left(E\left\|T^{+} B_{n 3}\right\|^{4}\right)^{1 / 2}=O\left(\frac{\delta^{2}}{a_{n}^{2}}\right) .
\end{gathered}
$$

See (6.11), (6.13), (6.14), and (6.15) of HH [11]. Moreover,

$$
\begin{aligned}
E \| & (I+T \Delta)^{-1} T^{+} \Delta T^{+} B_{n 22} \|^{2} \\
& =O\left(\frac{h^{2 r-1}}{n a_{n}^{2+(\alpha+1) / \alpha}}+\frac{1}{n^{3} h^{5} a_{n}^{4}}+\frac{h^{4 r}}{n h a_{n}^{2}}\right) .
\end{aligned}
$$

See the arguments leading to (6.24) in $\mathrm{HH}$ [11] and the analogous result for their equation (6.24) in $\mathrm{HH}$ [11] and the analogous result for their quantity $E\left\|H_{n 2}\right\|^{2}$. Combining (A.25)-(A.30) with Assumptions 5 and 7 yields the result that

$$
\frac{A_{n 4}(x, z)}{\sqrt{V_{n}(x, z)}}=\frac{-\left(I+T^{+} \Delta\right)^{-1} T^{+} B_{n 12}}{\sqrt{V_{n}(x, z)}}+o_{p}(1) .
$$

Now consider $-\left(I+T^{+} \Delta\right)^{-1} T^{+} B_{n 12}$. Standard calculations for kernel estimators show that

$$
\begin{gathered}
\iiint_{0}^{1} \widehat{f}_{\mathbb{X} \mathbb{Z} W}(x, z, w) f_{\mathbb{X} \mathbb{Z} W}(x, z, w) g(x, z) d x d z d w \\
=n^{-1} \sum_{i=1}^{n} f_{\mathbb{X} \mathbb{Z} \mathbb{W}}\left(x, z, \mathbb{W}_{i}\right) g\left(\mathbb{X}_{i}, \mathbb{Z}_{i}\right)+O\left(h^{r}\right) .
\end{gathered}
$$

Therefore,

$$
\begin{aligned}
& T^{+} \iiint_{0}^{1} \widehat{f}_{\mathbb{X} \mathbb{Z} \mathbb{W}}(x, z, w) f_{\mathbb{X} \mathbb{Z} \mathbb{W}}(x, z, w) g(x, z) d x d z d w \\
& \quad=A_{n 1}(x, z)+o\left(\frac{h^{r}}{a_{n}}\right),
\end{aligned}
$$

$$
T^{+} B_{n 12}(x, z)=A_{n 1}(x, z)-E A_{n 1}(x, z)+o\left(\frac{h^{r}}{a_{n}}\right) .
$$

But

$$
\begin{aligned}
(I & \left.+T^{+} \Delta\right)^{-1} T^{+} B_{n 12}(x, z) \\
& =T^{+} B_{n 12}+\left[\left(I+T^{+} \Delta\right)^{-1}-I\right] T^{+} B_{n 12} \\
& =T^{+} B_{n 12}+\left(\widehat{T}+a_{n} I\right)^{-1} \Delta T^{+} B_{n 12}
\end{aligned}
$$

Therefore, it follows, by combining Assumption 7 and equations (A.11), (A.17), and (A.34), that

$$
\left(I+T^{+} \Delta\right)^{-1} T^{+} B_{n 12}(x, z)=A_{n 1}(z)-E A_{n 1}(x, z)+r_{n}
$$

where $E\left\|r_{n}\right\|^{2} / \sqrt{V_{n}(x, z)}=o(1)$ for almost every $(x, z)$. Combining this result with (A.21) and (A.31) gives

$$
\frac{A_{n 3}(x, z)}{\sqrt{V_{n}(x, z)}}=\frac{-\left[A_{n 1}(x, z)-E A_{n 1}(x, z)\right]}{\sqrt{V_{n}(x, z)}}+o_{p}(1)
$$

for almost every $(x, z)$.

Now consider $A_{n 4}(x, z)$. HH show that

$$
A_{n 4}(x, z)=-\left(I+T^{+} \Delta\right)^{-1} T^{+} \Delta\left(A_{n 2}-T^{+} B_{n 2}\right)(x, z) .
$$

Therefore, it follows from (A.19) and (A.30) that

$$
\frac{A_{n 4}(x, z)}{\sqrt{V_{n}(x, z)}}=o_{p}(1)
$$

for almost every $(x, z)$.

Now combine (A.19), (A.37), and (A.39) to obtain

$$
\frac{S_{n 2}(x, z)}{\sqrt{V_{n}(x, z)}}=\frac{\sum_{j=1}^{4} A_{n j}(x, z)}{\sqrt{V_{n}(x, z)}}=\frac{E A_{n 1}(x, z)}{\sqrt{V_{n}(x, z)}}+o_{p}(1)
$$

for almost every $(x, z)$.The lemma follows by combining this result with (A.16).

This completes the proof.

\section{Conflict of Interests}

The author declares that there is no conflict of interests regarding the publication of this paper. 


\section{References}

[1] F. Black and M. Scholes, "The pricing of options corporate liabilities," Journal of Political Economy, vol. 81, pp. 637-659, 1973.

[2] R. C. Merton, "Theory of rational option pricing," Bell Journal of Economics and Management Science, vol. 4, no. 1, pp. 141-183, 1973.

[3] F. Antonelli, "Backward-forward stochastic differential equations," The Annals of Applied Probability, vol. 3, no. 3, pp. 777793, 1993.

[4] H. Wang, W. Li, and X. Wang, "Asymptotic stabilization by state feedback for a class of stochastic nonlinear systems with timevarying coefficients," Mathematical Problems in Engineering, vol. 2014, Article ID 258093, 6 pages, 2014.

[5] W. Zhang and G. Li, "Discrete-time indefinite stochastic linear quadratic optimal control with second moment constraints," Mathematical Problems in Engineering, vol. 2014, Article ID 278142, 9 pages, 2014.

[6] Y. Su and L. Lin, "Semi-parametric estimation for forwardbackward stochastic differential equations," Communications in Statistics: Theory and Methods, vol. 38, no. 11, pp. 1759-1775, 2009.

[7] X. Chen and L. Lin, "Nonparametric estimation for FBSDEs models with applications in finance," Communications in Statistics-Theory and Methods, vol. 39, no. 14, pp. 2492-2514, 2010.

[8] L. Lin, F. Li, and L. X. Zhu, "On regression with variance built-in mean regression function: a new financial model," Manuscript, 2009.

[9] Q. Zhang and L. Lin, “Terminal-dependent statistical inferences for FBSDE," Stochastic Analysis and Applications, vol. 32, pp. 128-151, 2014.

[10] C. Hsiao, Analysis of Panel Data, vol. 36 of Econometric Society Monographs, Cambridge University Press, Cambridge, UK, 2nd edition, 2003.

[11] P. Hall and J. L. Horowitz, "Nonparametric methods for inference in the presence of instrumental variables," The Annals of Statistics, vol. 33, no. 6, pp. 2904-2929, 2005.

[12] A. N. Kolmogorov and U. A. Rozanov, "On the strong mixing conditions of a stationary Gaussian process," Theory of Probability and Its Applications, vol. 2, pp. 222-227, 1960.

[13] R. C. Bradley and W. Bryc, "Multilinear forms and measures of dependence between random variables," Journal of Multivariate Analysis, vol. 16, no. 3, pp. 335-367, 1985.

[14] C. R. Lu and Z. Y. Lin, Limit Theories for Mixing Dependent Variables, Science Press, Beijing, China, 1997.

[15] D. N. Politis and J. P. Romano, "A general resampling scheme for triangular arrays of $\alpha$-mixing random variables with application to the problem of spectral density estimation," The Annals of Statistics, vol. 20, no. 4, pp. 1985-2007, 1992. 


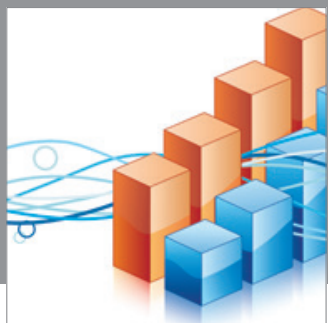

Advances in

Operations Research

mansans

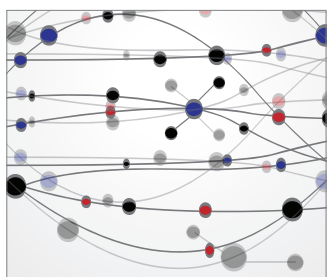

The Scientific World Journal
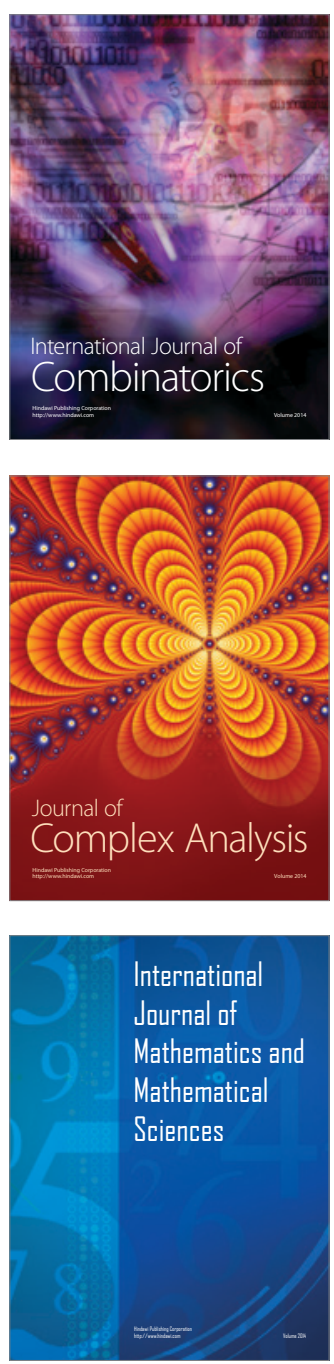
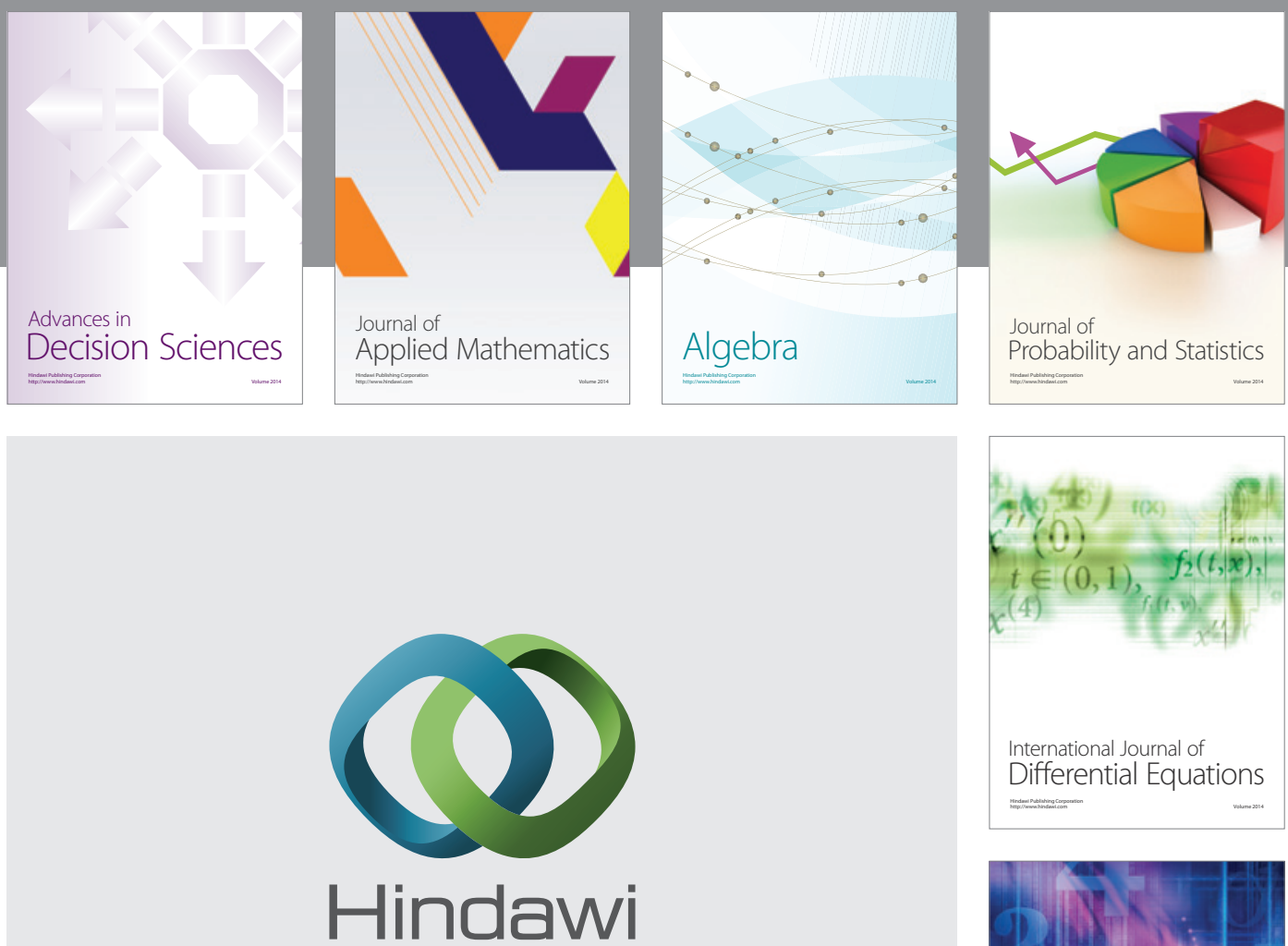

Submit your manuscripts at http://www.hindawi.com
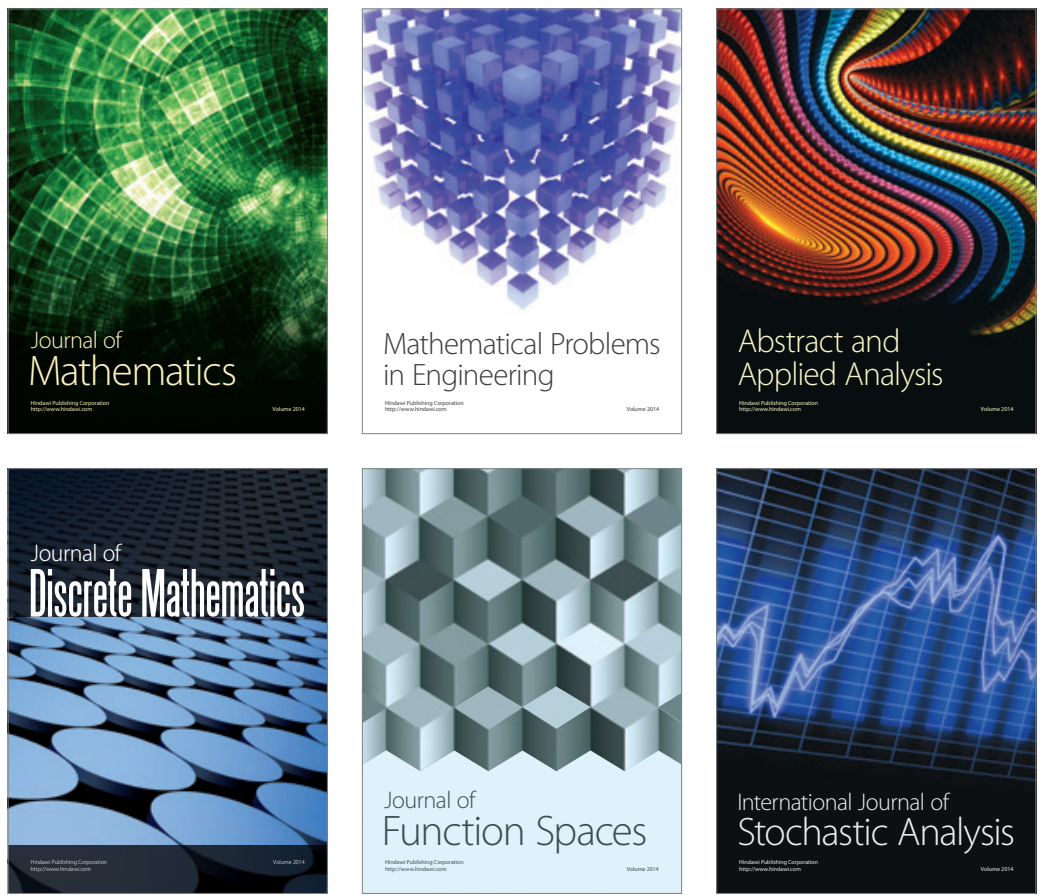

Journal of

Function Spaces

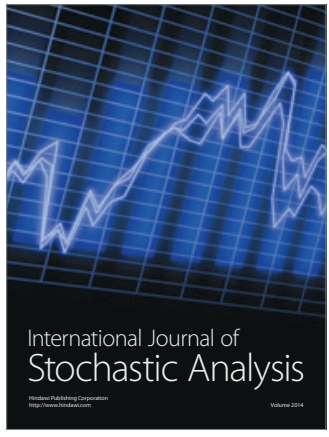

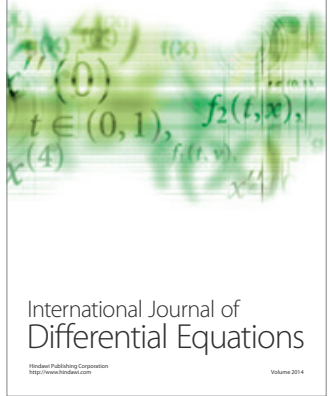
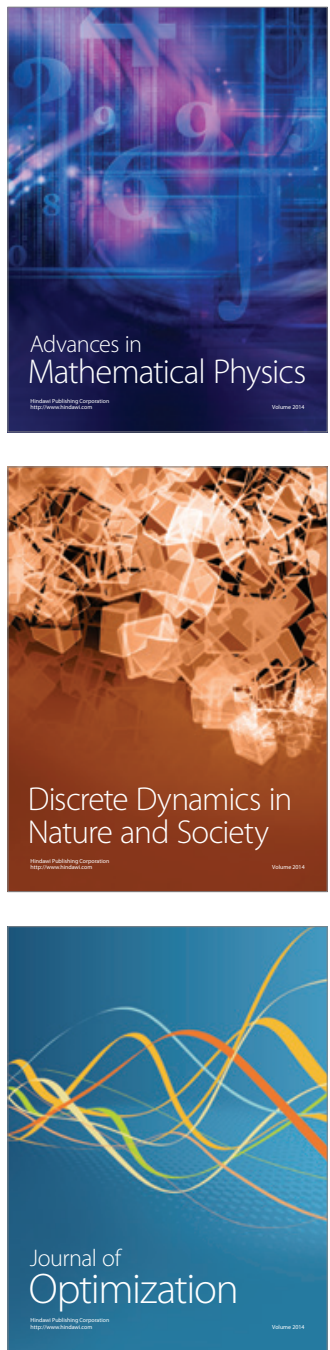